\title{
Salivary IgA as a Useful Non-Invasive Marker for Restraint Stress in Pigs
}

\author{
Yoshihiro MUNETA ${ }^{1 * *}$, Tadao YOSHIKAWA ${ }^{2 *}$, Yu MINAGAWA ${ }^{1)}$, Tomoyuki SHIBAHARA ${ }^{3)}$, Ryuichiro MAEDA ${ }^{2)}$ \\ and Yoshitaka OMATA ${ }^{2)}$ \\ ${ }^{1)}$ Research Team for Advanced Biologicals, National Institute of Animal Health, 3-1-5, Kannondai, Tsukuba, Ibaraki, 305-0856, \\ ${ }^{2)}$ Department of Basic Veterinary Science, Obihiro University of Agriculture and Veterinary Medicine, Obihiro, Hokkaido, 080-8555 and \\ ${ }^{3)}$ Epidemiological Research Team, National Institute of Animal Health, 3-1-5 Kannondai, Tsukuba, Ibaraki 305-0856, Japan
}

(Received 7 January 2010/Accepted 25 April 2010/Published online in J-STAGE 11 May 2010)

ABSTRACT. In this study, we investigated the expression of immunoglobulin A (IgA) in porcine salivary gland and its relationship with restraint stress in pigs. IgA was expressed in plasma cells in pig salivary gland, as confirmed by immunohistochemical staining. IgA was also detected in pig saliva itself by ELISA, and salivary IgA levels were increased by a restraint stress. Moreover, there was a circadian rhythm of IgA over the course of a day. These results are the first evidence of IgA expression related to stress in the pig saliva and may make IgA useful as a non-invasive biological marker to evaluate acute stress condition in the pigs. KEY WORDS: IgA, non-invasive biomarker, restraint stress, saliva, salivary glands.

Recently, interest in animal welfare in relation to the handling, housing, and feeding of domestic and companion animals has been expanding, and concern has been growing that increases in intensive agriculture and changes in environmental factors may enhance physiological stress in animals [5]. These stresses negatively affect resistance to diseases such as diarrhea [24] and pneumonia [17], as well as the meat quality [26] and reproduction [6] in a variety of animals. Recently, guidelines for the handling, housing, and feeding of pigs and laying chickens with regard to animal welfare have been introduced in Japan (http://jlta.lin.gr.jp/ chikusan/aw/aw.html). However, reliable, easy and noninvasive methods and biomarkers to evaluate stress in animals have not been established thus far.

Saliva sampling has the advantage of being non-invasive, anyone can easily perform it, and it is stress-free in comparison with blood sampling. Therefore, saliva is considered to be a good material for evaluating the stress condition, and salivary biomarkers to evaluate stress have received much attention in humans [28]. Several salivary markers related to stress have been reported, including cortisol [10, 13, 27], $\alpha$-amylase [19, 27], $\beta$-endorphin [21], and chromogranin A [11]. Salivary Immunoglobulin A (IgA) has also been reported to be a potential stress marker in rats [8], humans [30] and dogs [12]. However, few studies have been performed to evaluate salivary IgA as a stress marker in domestic animal species such as pigs. Most of the past research regarding stress markers in pigs has been carried out with blood samples such as with the measurement of serum cortisol levels [1], leukocyte populations and functions such as natural killer cytotoxicity [20] or long-term observation of stress-related activity such as tail biting [23]. Therefore, a

\footnotetext{
* Correspondence to: Muneta, Y., Research Team for Advanced Biologicals, National Institute of Animal Health, 3-1-5 Kannondai, Tsukuba, Ibaraki 305-0856, Japan. e-mail: ymuneta@affrc.go.jp

\# Equal contributions.
}

non-invasive biomarker in pigs should be established to eliminate unnecessary stress and to improve animal welfare status as well as to increase productivity.

Therefore, in the present study, we investigated the expression of $\operatorname{Ig} \mathrm{A}$ in pig salivary gland and saliva, and examined its dynamics with an acute restraint stress to evaluate IgA as a non-invasive stress marker in pigs.

\section{MATERIALS AND METHODS}

Animals: Eight LW F1 male piglets (6-8 weeks olds) were used in this experiment. They were kept in an animal room $(4.8 \times 3.7 \times 2.4 \mathrm{~m})$ with natural light from a glass window, which was shut all the time to avoid any sounds, dust, etc. They were fed with normal feeding for growing pigs at 8:00 and 18:00 with a free water supply. All animal experiments were approved by the committee for animal experiments of Obihiro University of Agriculture and Veterinary Medicine (No. 18-59). The salivary gland samples for the immunohistochemistry of IgA were taken from 4 healthy LWD pigs (5-month olds) at the National Institute of Animal Health.

IgA expression in pig salivary glands by immunohistochemistry: Salivary glands (parotid gland, submandibular gland, and sublingual gland, respectively) were fixed in Zamboni fixative (Kyodo Byori Inc., Kobe, Japan) overnight at $4^{\circ} \mathrm{C}$ and embedded in paraffin as described previously [18]. Paraffin sections were immunostained using $1 \mathrm{mg} / \mathrm{ml}$ of goat anti-pig IgA antibody (1:4,096 working dilution, Bethyl Laboratories Inc., Montgomery, TX, U.S.A.) with Histofine simple stain MAX-PO (G) solution (Nichirei Corp., Tokyo, Japan). Normal goat serum was used as a negative control. The number of plasma cells that had positively stained in each salivary gland was estimated by microscopic observation using $200 \times$ magnification. The number of plasma cells in twenty randomly selected microscopic fields in each section was counted and the mean was calculated. 
(A)

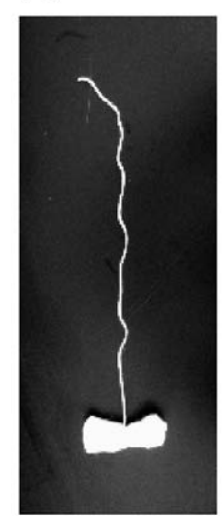

(B)

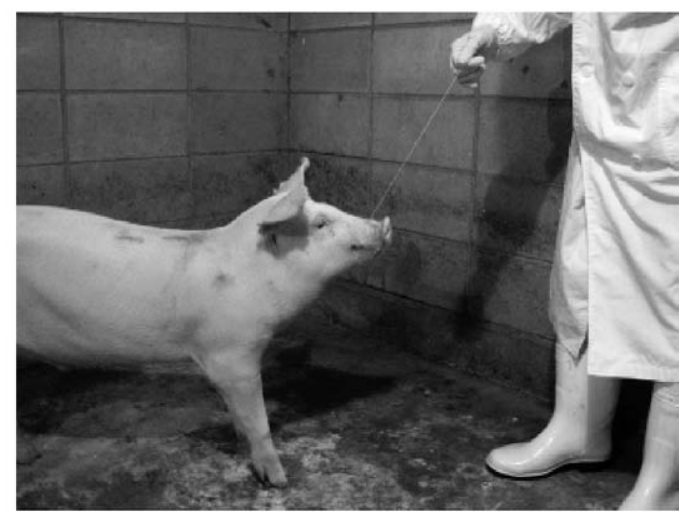

Fig. 1. Method for the collection of pig saliva. (A) Tools for saliva collection. (B) A picture of collection of saliva from a pig.

Saliva collection: Saliva samples were collected by the following methods. Medical absorbent cotton $(4 \times 5 \mathrm{~cm})$ was prepared and tied with $50 \mathrm{~cm}$ of string (Fig. 1A). It was placed in front of a pig and was allowed to be bitten by the pigs (Fig. 1B). After $2 \mathrm{~min}$, the cotton was recovered by withdrawing the strings and put in a $10-\mathrm{m} l$ syringe. The syringe containing the cotton was put in another $50-\mathrm{m} l$ tube and kept on ice. Saliva was collected by centrifugation at $2,500 \mathrm{~g}$ for $15 \mathrm{~min}$, and the collected saliva was kept $-30^{\circ} \mathrm{C}$ until analysis. Five- to one-hundred microliters of saliva without any other treatment was used for the following assays to evaluate salivary stress markers.

Assays: In this study, salivary IgA, cortisol and $\beta$-endorphin concentration as well as $\alpha$-amylase activity were measured by the following assays. IgA concentrations were determined by ELISA using a PIG IgA ELISA quantitation kit (Bethyl Laboratories Inc.) as described by the manufacturer's instruction, which contains goat anti-pig IgA affinity purified antibody as a coating antibody and HRP-conjugated goat anti-pig IgA as a detection antibody. Range of detection for this ELISA is $15.625-1,000 \mathrm{ng} / \mathrm{ml}$. One hundred-times diluted saliva was used as a sample. Cortisol levels were measured by a competitive ELISA in the same saliva using a goat anti-mouse IgG $(\mathrm{H}+\mathrm{L})$ (Vector Laboratories Inc., Burlingame, CA, U.S.A.) as a primary capture antibody and anti-cortisol mouse monoclonal antibody (Chemicon International, Temecula, CA, U.S.A.) as a secondary capture antibody, and hydrocortisones and HRPlabeled hydrocortisones (Sigma Chemical Co., St. Louis, MO, U.S.A.) as a standard and competitor, respectively. Undiluted saliva was used as a sample. We also measured $\alpha$-amylase activity with the salivary $\alpha$-amylase assay kit (Salimetrics LLC, State College, PA, U.S.A.) which is available for the measurement of pig $\alpha$-amylase activity (personal communication with technical support of Salmetrics) and $\beta$-endorphin concentrations by RIA using an anti$\beta$-endorphin rabbit anti-serum (Sigma Chemical Co.) and a ${ }^{125}$ I-labelled $\beta$-endorphin (Amersham Bioscience Co., Piscataway, NJ, U.S.A.). Ten-times diluted saliva for $\alpha$-amy- lase and undiluted saliva for $\beta$-endorphin was used as a sample, respectively.

Circadian rhythm of IgA levels in pig saliva: Four piglets were used to determine the circadian rhythm of salivary IgA. Saliva samples were taken from each piglet at 09:00, 11:00, 13:00, 15:00, and 17:00, respectively. Samples were collected and treated as described above. Three experiments with the same time schedule were repeated on 3 different days at 1-day intervals. IgA concentrations in each saliva sample were determined by ELISA using a PIG IgA ELISA quantitation kit (Bethyl Laboratories Inc.) as described above.

Changes in IgA levels in response to restraint stress: Eight pigs were subjected to a restraint stress. Restraint was started at 9:00. At first, saliva samples were collected to wash the oral cavity and discarded. After $5 \mathrm{~min}$, pre-stress (0 min) saliva samples were collected. Then, the pigs were subjected to a restraint stress for $20 \mathrm{~min}$ and saliva was collected 10 and $20 \mathrm{~min}$ after the start of restraint. For the restraint, pigs were taken to the corner of the room and immobilized within $120 \times 35 \times 70 \mathrm{~cm}$ of space by 2 iron fences. In this situation, pigs were not able to move, as the side of the head and one side of the body were attached to a concrete wall, and the hip side and the other body side were attached to 2 iron fences. The pigs were then released from the restraint and allowed to walk freely, and the saliva was collected again $10 \mathrm{~min}$ after the end of restraint. Control salivary samples were collected from the same pigs on another day with the same time-interval without restraint stress. $\operatorname{IgA}$, cortisol and $\beta$-endorphin concentration as well as $\alpha$ amylase activity in each saliva sample were determined as described above.

Data and statistical analysis: The data of IgA, cortisol and $\alpha$-amylase was represented by increase rate which was calculated pre-stress concentration as $100 \%$ because of the individual variability. $\beta$-endorphin concentration was presented by actual measured values as some of the pre-stress saliva contains no or very few $\beta$-endorphin. The differences between each group or time point were analyzed using the 
(A) Parotid gland

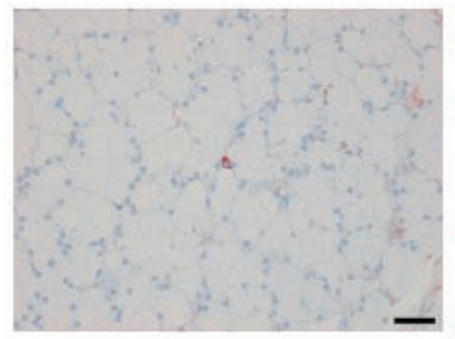

(D) Sublingual gland

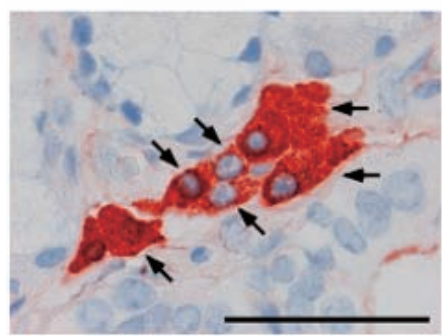

(B) Submandibular gland

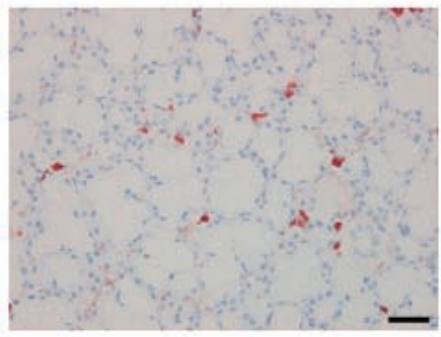

(E) Number of IgA-positive cells in each salivary gland

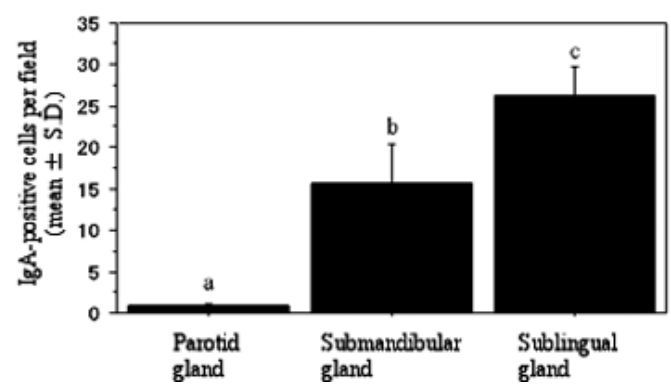

Fig. 2. Immunohistochemical staining of IgA in pig salivary gland. (A) Parotid gland, (B) Submandibular gland, (C) Sublingual gland, and (D) High magnification of sublingual gland. Each picture is a representative picture of a similar pattern of staining from four different pigs. Arrows show IgA-positive plasma cells. Bar=50 $\mu \mathrm{m}$. (E) The number of IgA-positive plasma cells in each salivary gland. The number of IgA-positive plasma cells was counted as described in Materials and Methods. There are significant difference among different superscript letters $(P<0.05)$.

repeated measure ANOVA test or Student's $t$-test in Statview (Abacus Concept, Berkeley, CA, U.S.A.). $P<0.05$ was considered to be significant.

\section{RESULTS}

IgA expression in pig salivary glands by immunohistochemistry: IgA was detected in the cytoplasm of plasma cells in the salivary gland by immunohistochemistry, as shown in Fig. 2A-D. The sublingual gland showed the highest expression level, then the submandibular gland, and the lowest was the parotid gland (Fig. 2C, 2B and 2A, respectively). In accord with the results of observation by microscopy, the number of IgA-positive plasma cells was also significantly different in each salivary gland (Fig. 2E).

IgA levels in pig saliva: IgA was also detected in saliva by ELISA, as shown in Fig. 3. IgA levels in saliva were approximately $10-70 \mu \mathrm{g} / \mathrm{m} l$. There were significant changes for the IgA levels to show a circadian rhythm throughout the day, being low in the morning (9:00), rising in the daytime (11:00-15:00), and then decreasing in the evening (17:00).

Changes in IgA levels in response to restraint stress: Figure $4 \mathrm{~A}$ illustrates the changes in $\mathrm{IgA}$ during restraint stress. Salivary IgA levels were significantly increased after 10 min of restraint stress and remained high after $20 \mathrm{~min}$ $(P<0.01)$. However, IgA levels returned to pre-stress levels

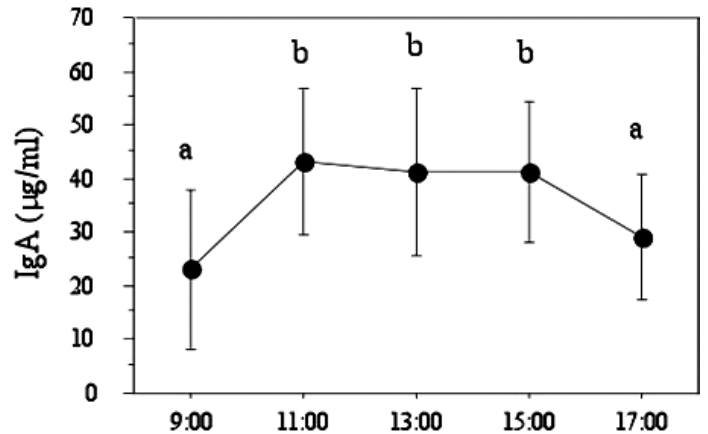

Fig. 3. Circadian rhythm of IgA levels in pig saliva. Data show the mean \pm SD of twelve measurements from three experiments on three different days using four different pigs. There are significant difference between $\mathrm{a}$ and $\mathrm{b}$ $(P<0.05)$.

10 min after the restraint stress ended.

Dynamics of other stress markers: We also measured cortisol as a conventional stress marker in saliva during the restraint stress. As shown in Fig. 4B, saliva cortisol levels were also significantly increased after the restraint stress $(P<0.01)$. However, the increase rate was lower than that of $\operatorname{IgA}$, and the increases in cortisol continued for more than 10 min after the end of the stress.

In contrast, the $\alpha$-amylase activities in saliva showed no 
relation to the stress, and $\beta$-endorphin levels were increased after the stress, but no significant difference was observed due to the large variation among the samples (Fig. 4C and 4D).

\section{DISCUSSION}

In this study, we examined the expression of IgA in porcine salivary gland and its dynamics during a restraint stress in pigs to show the usefulness of $\operatorname{IgA}$ as a non-invasive stress marker. Restraint stress sometimes occurs in pig production. For example, when blood samples are taken from pigs for diagnosis or treatment, pigs must be immobilized using a pig keeper or some other device. However, the saliva collection carried out in this study was easy, non- invasive, and relatively stress-free. Moreover, anyone can carry out the sampling by this method without a special apparatus or techniques in comparison with blood sampling. Therefore, stress evaluation using saliva samples are straightforward in relation to animal welfare issues.

Indeed, IgA was detected in pig salivary glands by immunohistochemistry. In the salivary gland, IgA was immunostained in the cytoplasm of plasma cells. Interestingly, the number of plasma cells found in each salivary gland was different among parotid, submandibular, and sublingual glands. The secretions of water and saliva component are controlled by parasympathic and sympathic nerve systems (PNS and SNS). The parotid gland secretes mainly fluid and electrolyte components of saliva and is regulated by PNS impulses [7]. The submandibular and sublingual

\section{(A) IgA}

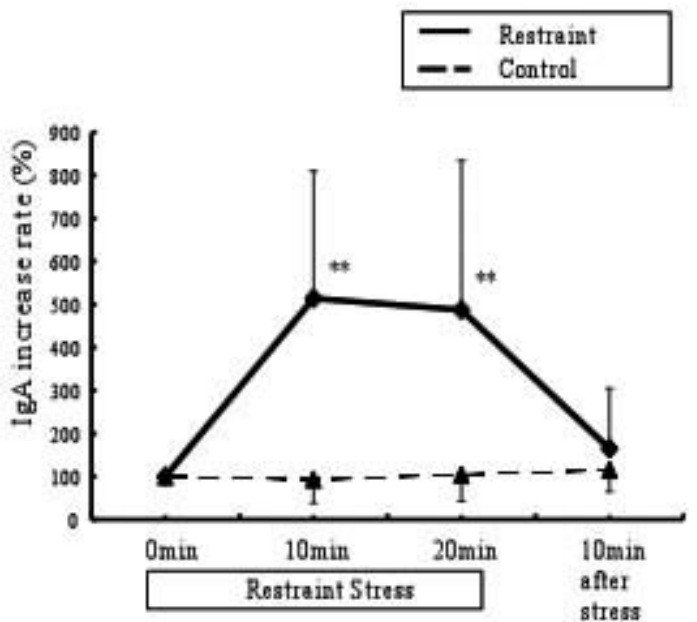

(C) $\alpha$-amylase

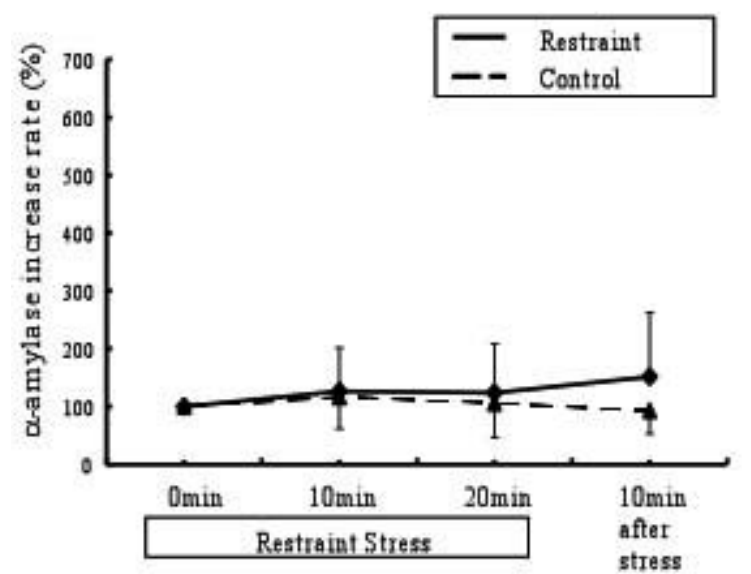

(B) Cortisol

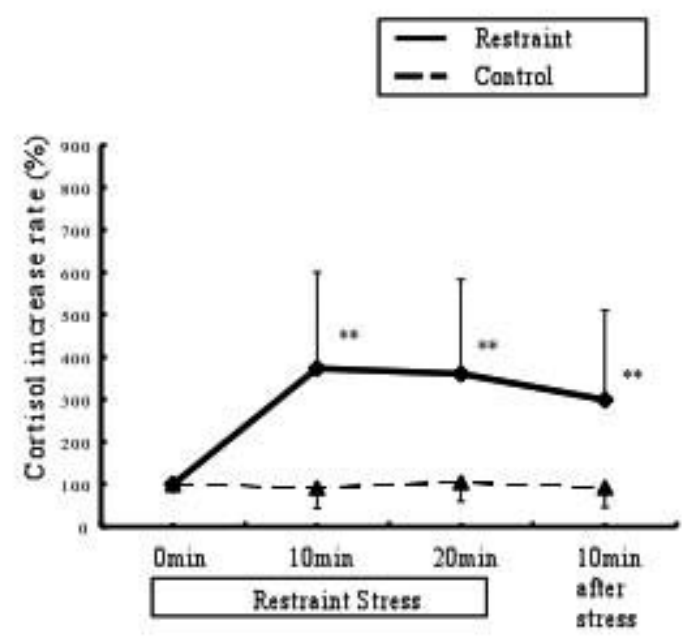

(D) $\beta$-endorphin

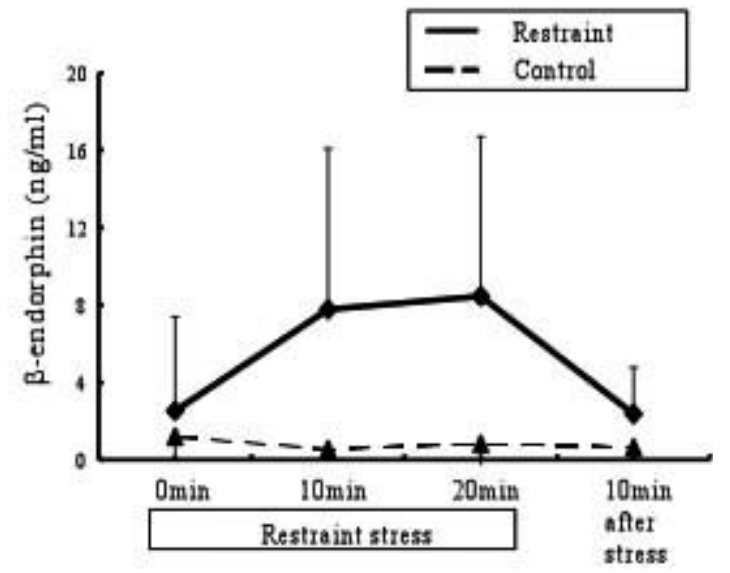

Fig. 4. The changes in salivary IgA, cortisol, $\alpha$-amylase and $\beta$-endorphin during a restraint stress in pigs. (A) IgA, (B) Cortisol, (C) $\alpha$-amylase and (D) $\beta$-endorphin. Data show the mean \pm SD from eight different pigs. Control data was obtained from the same eight pigs without restraint on different days. ${ }^{* *}$ shows significant difference $(P<0.01)$. 
glands are mixed glands, and secretion of the protein component of saliva is enhanced by SNS impulses [22]. Carpenter et al., reported that the secretion of IgA into saliva was affected by a polymeric immunoglobulin receptor (pIgR)mediated mechanism in response to autonomic stimulation $[3,4]$, and parotid cells and submandibular cells were differently responded to alpha- and beta-adrenergic stimuli in rat model [4]. Taken together, it is reasonable to assume that IgA secretion in response to a stress signal derived from the activation of SNS would be primarily observed in the submandibular and sublingual glands, although further studies must be performed using pig salivary glands to reveal the relationship salivary IgA secretion and autonomic stimulation in stress response in pigs.

In addition, IgA was detected in saliva itself by ELISA. The IgA levels in normal healthy pigs were found to be approximately $10-70 \mu \mathrm{g} / \mathrm{ml}$ in this study. A circadian rhythm of IgA was observed throughout the day, as has been reported in dogs [12]. We would suggest that the time for saliva sampling as well as feeding should be considered when using $\operatorname{IgA}$ as a marker for stress evaluation.

During the restraint stress, salivary IgA levels were significantly increased. Previous studies showed that salivary IgA levels were decreased by a social stress in rat [8] and noise stress in dog [12], but increased by acute and cumulative multi-tasking stress in human [30]. The restraint stress performed in this study belong acute physiologic stress, so the activation of SNS stimuli was more faster and stronger than psychological stress such as social and noise stress, this may lead to the different salivary IgA dynamics after the stress.

After increasing by an acute restraint stress, salivary IgA levels were returned to pre-stress levels soon after removal of the stress. However, cortisol levels were still significantly increased after the removal of stress. This difference may be explained that $95 \%$ of salivary IgA is derived from salivary glands and stored in plasma cells as protein and released locally by the SNS stimuli of stress $[2,15]$, while cortisol is a hormone derived from adrenal cortex through blood by the activation of hypothalamo-pituitary-adrenocortical (HPA) axis [16]. Moreover, previous reports suggested that salivary cortisol is highly variable and is responsive to a wide range of factors, and requires certain methodological prerequisites $[9,29]$, but salivary secretory $\operatorname{IgA}$ is abundant in saliva and also exists as different form with serum $\operatorname{IgA}$ [15], and commercial kits for the specific measurement are available even in the domestic animal species such as pigs. Furthermore, the increase rate of $\mathrm{IgA}$ was comparable or better than cortisol, and the sample volume to use in the ELISA was 20-times less in IgA compared with cortisol (5 $\mu l$ for IgA vs $100 \mu l$ for cortisol). Taken together, we concluded that IgA is a better stress marker compared with cortisol in pig saliva.

Salivary $\alpha$-amylase was not related to restraint stress in this study. The concentrations of $\alpha$-amylase in saliva seem to be different and are affected by foods consumed by each species of animals, for example $\alpha$-amylase activities in dog and cat saliva has been reported very low $[14,25]$, so it is difficult to use as a stress marker in domestics animals. $\beta$ endorphin tends to be related to stress, but no significant results were obtained due to the high variability, so further studies must be carried out to evaluate $\beta$-endorphin as a salivary stress marker in pigs.

In conclusion, the data described herein provide the first evidence of the usefulness of salivary secretory IgA as a marker for an acute stress in pigs. Salivary IgA in pigs was better biomarker compared with cortisol and immediately returned to pre-stress levels after the end of the acute restraint stress. These results suggest that non-invasive measurement of salivary IgA may be one of a promising method for monitoring stress conditions in pigs.

ACKNOWLEDGMENTS. We dedicate this work to Professor Yoshitaka Omata. This work was partly supported by a grant for a healthy and safe pig industry project (1132) from the Ministry of Agriculture, Forestry and Fisheries of Japan.

\section{REFERENCES}

1. Becker, B. A., Nienaber, J. A., Christenson, R. K., Manak, R. C., DeShazer, J. A. and Hahn, G. L. 1985. Peripheral concentrations of cortisol as an indicator of stress in the pig. Am. J. Vet. Res. 46: 1034-1038.

2. Carpenter, G. H., Garrett, J. R., Hartley, R. H. and Proctor, G. B. 1998. The influence of nerves on the secretion of immunoglobulin A into submandibular saliva in rats. J. Physiol. 512: 567-573.

3. Carpenter, G. H., Proctor, G. B., Ebersole, L. E. and Garrett, J. R. 2004. Secretion of IgA by rat parotid and submandibular cells in response to autonomimetric stimulation in vitro. Int. Immunopharmacol. 4: 1005-1014.

4. Carpenter, G. H., Proctor, G. B. and Garrett, J. R. 2005. Preganglionic parasympathectomy decreases salivary SIgA secretion rates from rat submandibular gland. J. Neuroimmunol. 160: 4-11.

5. Caporale, V., Alessandrini, B., Dalla Villa, P. and Del Papa, S. 2005. Global perspectives on animal welfare: Europe. Rev. Sci. Tech. 24: 567-577.

6. Einarsson, S., Brandt, Y., Lundeheim, N. and Madej, A. 2008. Stress and its influence on reproduction in pigs: a review. Acta. Vet. Scand. 50: 48.

7. Garrett, J. R. 1987. The proper role of nerves in salivary secretion: a review. J. Dent. Res. 66: 387-397.

8. Guhad, F. A. and Hau, J. 1996. Salivary IgA as a marker of social stress in rats. Neurosci. Lett. 27: 137-140.

9. Hanrahan, K., McCarthy, A. M., Kleiber, C., Lutgendorf, S. and Tsalikian, E. 2006. Strategies for salivary cortisol collection and analysis in research with children. Appl. Nurs. Res. 19: 95-101.

10. Hellhammer, D. H., Wust, S. and Kudielka, B. M. 2009. Salivary cortisol as a biomarker in stress research. Psyconeuroendocrinology 34: 163-171.

11. Kanamaru, Y., Kikukawa, A. and Shimamura, K. 2006. Salivary chromogranin-A as a marker of psychological stress during a cognitive test battery in humans. Stress 9: 127-131.

12. Kikkawa, A., Uchida, Y., Nakade, T. and Taguchi, K. 2003. Salivary secretory IgA concentrations in beagle dogs. J. Vet. 
Med. Sci. 65: 689-693.

13. Kirschbaum, C. and Hellhammer, D. H. 1994. Salivary cortisol in psychoneuroendocrine research: recent development and applications. Psychoneuroendocrinology 19: 313-333.

14. McGeachin, R. L. and Akin, J. R. 1979. Amylase levels in the tissues and body fluids of the domestic cat (Felis catus). Comp. Biochem. Physiol. B. 63: 437-439.

15. Mestecky, J. 1988. Immunobiology of IgA. Am. J. Kidney Dis. 12: $378-383$.

16. Minton, J. E. 1994. Function of the hypothalamic-pituitaryadrenal axis and the sympathetic nervous system in models of acute stress in domestic farm animals. J. Anim. Sci. 72: 18911898.

17. Mitchell, G. B., Clark, M. E., Siwicky, M. and Caswell, J. L. 2008. Stress alters the cellular and proteomic compartments of bovine bronchoalveolar lavage fluid. Vet. Immunol. Immunopathol. 125: 111-125.

18. Nagai, Y., Watanabe, K., Aso, H., Ohwada, S., Muneta, Y. and Yamaguchi, T. 2006. Cellular localization of IL-18 and IL-18 receptor in pig anterior pituitary gland. Domest. Anim. Endocrinol. 30: 144-154.

19. Nater, U. M. and Rohleder, N. 2009. Salivary alpha-amylase as a non-invasive biomarker for the sympathetic nervous system: current state of research. Psychoneuroendocrinology 34: 486496.

20. Niekamp, S. R., Sutherland, M. A., Dahl, G. E. and SalakJohnson, J. L. 2007. Immune responses of piglets to weaning stress: impact of photoperiod. J. Anim. Sci. 85: 93-100.

21. Pikula, D. L., Harris, E. F., Desiderio, D. M., Fridland, G. H. and Lovelace, J. L. 1992. Methionine enkephalin-like, sub- stance P-like, and beta-endorphin-like immunoreactivity in human parotid saliva. Arch. Oral. Biol. 37: 705-709.

22. Sabbadini, E. and Berczi, I. 1995. The submandibular gland: a key organ in the neuro-immuno-regulatory network? Neuroimmunomodulation 2: 184-202.

23. Schroder-Petersen, D. L. and Simonsen, H. B. 2001. Tail biting in pigs. Vet. J. 162: 196-210.

24. Spencer, B. T. and Howell, P. G. 1989. Some husbandry factors influencing weaning stresses in piglets. J. S. Afr. Vet. Assoc. 60: 62-64.

25. Simpson, J. W., Doxey, D. L. and Brown, R. 1984. Serum isoamylase values in normal dogs and dogs with exocrine pancreatic insufficiency. Vet. Res. Commun. 8: 303-308.

26. Southern, K. J. Rasekh, J. G. Hemphil, F. E. and Thaler, A. M. 2006. Conditions of transfer and quality of food. Rev. Sci. Tech. 25: 675-684.

27. Takai, N., Yamaguchi, M., Aragaki, T., Eto, K., Uchihashi, K. and Nishikawa, Y. 2004. Effect of psychological stress on the salivary cortisol and amylase levels in healthy young adults. Arch. Oral Biol. 49: 963-968.

28. Yamaguchi, M. 2007. Stress evaluation using a biomarker in saliva. Nippon Yakurigaku Zasshi 129: 80-84 (in Japanese).

29. Weibel, L. 2003. Methodological guidelines for the use of salivary cortisol as biological marker of stress. Presse. Med. 32: 845-851.

30. Wetherell, M. A., Hyland, M. E. and Harris, J. E. 2004. Secretory immunoglobulin A reactivity to acute and cumulative acute multi-tasking stress: relationships between reactivity and perceived workload. Biol. Psychol. 66: 257-270. 